\title{
Experiencia de articulación e integración en prácticas de docencia, investigación y extensión en educación superior
}

\section{Articulation and integration experience in teaching, research and extension practices in higher education}

\author{
Manuel Schneider* \\ https://orcid.org/0000-0001-9730-7655 \\ mschneider@ayv.unrc.edu.ar \\ Coordinador Consejo Social I Universidad \\ Nacional Río Cuarto | Argentina
}

\author{
Estefanía Bresso** \\ https://orcid.org/0000-0002-4420-7375 \\ estefabresso@gmail.com
}

Daniela Giuppone $\star \star \star$
https://orcid.org/0000-0002-2037-1162
giupponedaniela@gmail.com

Integrantes del Equipo del Consejo Social | Universidad Nacional Río Cuarto | Argentina

\section{RESUMEN}

En este artículo se presentan diferentes procesos transitados desde la Universidad Nacional Río Cuarto en relación al camino a la integralidad. Se mencionan las acciones e historia del Consejo Social Universitario, como actor central de vinculación y articulador de las prácticas de aprendizaje, investigación y extensión, dado que deben darse de manera contextualizada e inserta en la realidad que se atraviesa.

En tal sentido se avanza hacia la creación de mecanismos institucionales que den respuesta a las problemáticas, con programas institucionales. Se pretenden la integralidad, comprendida como la articulación de actores sociales y universitarios que conciben el hacer profundamente ligado al actual contexto socio-histórico.

Esta propuesta se encuadra dentro de los lineamientos generales acordados en el Estatuto de la Universidad Nacional de Río Cuarto, en donde se expresa que la Universidad concibe a la extensión como otra forma de construcción de conocimiento articulado al saber científico. La extensión deberá abocarse al desarrollo del conocimiento práctico, experiencial, profesional e inductivo. Este conocimiento deberá generarse de manera local, transdisciplinarmente y tener finalidades prácticas, promoviendo la articulación y mutuo enriquecimiento de saberes.

PALABRAS CLAVE articulación, integralidad, docencia, investigación, extensión, consejo social, universidad, impacto territorial, experiencias. 


\section{ABSTRACT}

KEY WORDS

articulation, comprehensiveness, teaching, investigation, extension, social council, university, territorial impact, experiences.
This article presents different processes run at Universidad Nacional de Rio Cuarto in relation to the way of integrality. History and actions of the Consejo National Universitario, como actor central de vinculacion and articulating learning practices, research and extension services are described. These interactions must occur under a context and inserted in the currying reality.

In this sense, some progress its done creating institutional mechanisms able to give answers to the problems using special programs. Integrality is pretended understanding articulations of social and academical actors that conceive the tasks deeply connected in the actual social and historical contest.

This purpose is framed within the general guidance agreed in the Universidad Nacional de Rio Cuarto Statuto where extension is seen as another form of knowledge construction interacting with the scientific practice. Extension must advocate for developing practical-experiencial-professional and inductive knowledge. This knowledge must be generated locally and transdisciplinary with practical endpoints stimulating positive interactions and enrichment the body of knowledges. 


\section{PRESENTACIÓN}

En la siguiente presentación se pretende dar cuenta de diferentes recorridos transitados desde la Universidad Nacional Río Cuarto, en relación a procesos de extensión e integralidad en el sistema de educación superior.

En una primera instancia se mencionan experiencias que fueron generando antecedentes o el pasaje hacia la extensión y a prácticas universitarias integrales. Posteriormente se hace mención a algunos conceptos centrales a tener en cuenta al referirnos a integralidad. Avanzando en el desarrollo, se hace mención al contexto actual de emergencia sanitaria por SARS-CoV-2 y al proyecto de Más Impacto Territorial (+ IT), como respuesta de integralidad desarrollada de la UNRC ante esta nueva realidad.

Para finalizar se plantean nuevos interrogantes y desafíos como propuestas para futuras reflexiones.

\section{ANTECEDENTES DE ARTICULACIÓN}

En el año 2009 la Universidad Nacional de Río Cuarto (UNRC) aprobó, mediante Resolución 322/2009 del Consejo Superior, la incorporación de prácticas socio-comunitarias (PSC) en una o más asignaturas de todas las carreras de la institución. La resolución normatiza e instituye a las prácticas socio comunitarias como obligatorias en todas las carreras de la universidad con el objetivo de dar respuestas a problemáticas de sectores vulnerables.

\section{En el año 2009 la Universidad Nacional de Río Cuarto (UNRC) aprobó, mediante Resolución 322/2009 del Consejo Superior, la incorporación de prácticas socio-comunitarias (PSC) en una o más asignaturas de todas las carreras de la institución.}

Cabe destacar que la UNRC fue la primera universidad nacional Argentina que institucionaliza esta experiencia, lo que fue reconocido con el Primer Premio Presidencial "Prácticas Educativas Solidarias en 
Educación Superior 2010"; le han seguido en esta iniciativa, con diversas modalidades, otras Universidades. En la UNRC cada facultad fue implementándolas por diferentes modalidades, destacándose Medicina Veterinaria, donde las PSC se abordan desde el ingreso hasta el sexto año, se hace todo un trayecto de prácticas socio comunitarias y no es sólo una asignatura (Universidad Nacional de Río Cuarto, 2016).

La ley de Educación Superior (1994) establece en su ARTíCULO 56. -Los estatutos podrán prever la constitución de un Consejo Social Universitario (CSU), en el que estén representados los distintos sectores e intereses de la comunidad local, con la misión de cooperar con la institución universitaria en su articulación con el medio en que está inserta. Podrá igualmente preverse que el CSU esté representado en los órganos colegiados de la institución. (Ministerio de Justicia y Derechos Humanos, 1995)

EI CSU de la UNRC, inició su derrotero en 2007, ese año se convocó a Asamblea Universitaria para modificar el estatuto, pero no se llega a ningún acuerdo, levantándose la asamblea. En los proyectos planteados, uno de ellos, presentados por el claustro estudiantil, planteaba la incorporación de la figura del CSU. En 2010 se concreta la Asamblea Universitaria para la modificación del estatuto que incluye la conformación del Consejo Social, mediante el TÍTULO IV -Consejo Social ARTICULO 38: El Consejo Social tendrá como misión entender en todo lo referente a la relación, vinculación e implementación de acciones conjuntas, directas y permanentes entre esta Universidad Nacional y la comunidad local y/o regional. En 2014: se reglamenta y se instruye la creación del Consejo Social a partir del Consejo Superior de la UNRC (UNRC, 2011)

Otra de las acciones institucionales que acompañan el proceso comenzado por el CSU son, en el año 2015, la generación de las mesas de prácticas socio comunitarias, se recuperan las PSC que habían tenido un intervalo, y se genera la Universidad Barrial (UB) en el 2015, en nuestra universidad. Mencionamos la UB, porque después, si hay tiempo, recuperamos, porque son las grietas, los bypass que tiene el sistema como para poder dar respuesta rápida, que es uno de los problemas que tienen estas actividades, o sea, los tiempos de la universidad y los tiempos de la comunidad suelen estar desfasados y entran en tensiones.

\section{EL TRAYECTO DEL CONSEJO SOCIAL UNIVERSITARIO}

Como se mencionó anteriormente a partir del 2014 se dispone la creación del CSU y se conforma una comisión que elabora la reglamentación, dependiente de la Secretaría de Extensión de la UNRC. Durante el 2014 se logra modificar el esquema de "Prioridades en Ciencia y Técnica" mediante la Resolución del Consejo Superior 137/2014 en el cual se incluye como líneas estratégicas las que propongan instituciones y organizaciones que conforman el CSU, que año a año ratifica el 
órgano superior. (UNRC, 2014) (UNRC, 2015).

Entre el 2015-2016 se designa al coordinador del CSU y se comienzan a generar relevamientos de organizaciones e instituciones, evaluando el vínculo que éstas mantenían con la UNRC y con un carácter de sistematizar esa información.

En el año 2017 asume una nueva la coordinación del CSU, se comienza a participar del Encuentro de Consejos Sociales, tomando como referencia los antecedentes del Consejo Social de la Universidad Nacional de La Plata, repensando, adaptamos, e intercambiando modos de trabajo y lineamientos generales, priorizando condiciones locales. Se realiza este año, conjuntamente con la Secretaría de Planeamiento, relevamientos cualitativos, mediante la técnica de la entrevista profunda a fin de ahondar en el conocimiento de propuestas, necesidad y demandas de 53 instituciones y organizaciones de Río Cuarto, en relación a la UNRC.

En 2018-2019, comenzamos a aprender junto con organizaciones e instituciones la idiosincrasia y a construir formas de trabajo locales-territoriales que se asientan en ejidos específicos de la ciudad. Estas formas de trabajo asumen la forma de Mesas Territoriales (MT). Las MT son espacios de trabajo que se organizan por barrios o por zonas, integradas por organizaciones e instituciones del lugar, entre ellas: escuelas en cualquiera de sus modalidades, copas de leches, merenderos, comedores, centros culturales, centros comunitarios, asociaciones civiles o asociaciones de hecho, clubes, vecinales, organizaciones sin fines de lucro, iglesias cualquiera sea su credo, asociaciones de interés, etc. Las MT son estructuras organizativas que están asentadas, en su mayoría, en sectores de pobreza estructural y se reúnen según lo dispongan sus integrantes. Se planifican propuestas, canalizar demandas, sugerir capacitaciones e ideas y también funciona como espacio de encuentro para los integrantes. Existe un objetivo implícito de organización territorial, generar espacios de organización a la comunidad, debatir, priorizar y generar proyectos, que en esos proyectos y esas demandas, la universidad pueda ir determinando en que puede acompañar, señalar los instrumentos y dispositivos con los que cuenta (desde la extensión, investigación, PSC, Universidad Barrial u otra instancia universitaria) y que se predisponga a la articulación y a la creación conjunta.

\section{En 2018-2019, comenzamos a aprender junto con organiza- ciones e instituciones la idiosincrasia y a construir formas de trabajo locales-territoriales que se asientan en ejidos especí- ficos de la ciudad.}

Por otra parte, también se cuenta con Mesa Temáticas, conforma-

das en base a Ejes Problemáticos o Temáticos propuestos por quien lo 
impulse al Consejo Social, los ejes o temas serán transversales y pueden ser tomados para el trabajo también por Mesas Territoriales.

Durante el 2020 las Mesas Territoriales han brindado certezas en la articulación con las estructuras que se han formado en el marco de la pandemia, o sea, la Universidad con las Mesas Territoriales son las que identifican problemáticas, proponen soluciones, articulan diagramando propuestas y, en conjunto se intenta la implementación y ejecución.

\section{AVANZANDO HACIA LA INTEGRALIDAD}

En el Plan Estratégico Institucional (PEI) 2017-2023, de nuestra casa de estudios, se plantea como Eje Estratégico № 4 el compromiso social mediante la construcción de redes territoriales, planteando que presupone la "democratización del conocimiento que ella produce y enseña mediante el desarrollo de prácticas académicas centradas en la intencionalidad de una mayor justicia e igualdad social. Significa también un proceso de apropiación social y distribución de saberes que se comparten, se recontextualizan en nuevos ámbitos y se ponen al servicio de la sociedad... El compromiso social se vincula también con la idea de territorializar la universidad; es decir, la universidad pasa a ser una institución más que configura la trama territorial; universidad y territorio comparten problemas y soluciones. El territorio pasa a ser, con sus múltiples dimensiones, el espacio donde se desenvuelve la vida universitaria".

El PEI también se basa en un modelo de universidad como espacio público donde, a través del conocimiento, la formación, la articulación social y la reflexión crítica contribuyen a pensar, comprender y transformar los diversos problemas de la sociedad que integra, en especial de aquellos que afectan a los sectores más vulnerables. De este modo, la calidad científica y académica se vincula con la función social de la Universidad que refiere a un proceso dinámico en el que universidad y sistema social se interpelan y transforman mutuamente en un contexto histórico concreto.

\section{EI PEI también se basa en un modelo de universidad como es- pacio público donde, a través del conocimiento, la formación, la articulación social y la reflexión crítica contribuyen a pen- sar, comprender y transformar los diversos problemas de la sociedad que integra, en especial de aquellos que afectan a los sectores más vulnerables.}

El compromiso social de la Universidad supone la democratización del conocimiento que ella produce y enseña mediante un proceso de apropiación social y distribución de saberes que se comparten, se re- 
contextualizan en nuevos ámbitos y se ponen al servicio de la sociedad. Más precisamente, el concepto de función social de la Universidad alude a: a) la respuesta a necesidades de los grupos sociales vulnerables, del estado y organizaciones de la sociedad civil; b) la producción, enseñanza y utilización de conocimientos científicos y tecnológicos contribuyendo a pensar y resolver los problemas críticos de la región; c) el establecimiento de relaciones de diálogo, intercambio y aprendizaje con actores sociales sobre problemáticas críticas comunes.

El paradigma neoliberal de formación de estudiantes y docentes con un perfil individualista y desentendido de las problemáticas de la sociedad, que observará su campo laboral-social de manera pragmática e instrumental. Ante ello proponemos una formación integral e íntegra, que capacite y constituya un estudiante que se reconozca inserto en un medio social que lo atraviesa, que lo interpela y lo exige a fin de poner en juego sus conocimientos académicos, su capacidad de planificación, su mirada política, su sensibilidad social, su creatividad laboral y su capacidad de autoempleo (Universidad Nacional de Rio Cuarto, 2016).

En este marco se considera de fundamental importancia la oportunidad que supone la existencia del CSU como apuesta de retroalimentación con organizaciones de la sociedad civil, ya que los mismos relevan las necesidades, de los diferentes barrios y las inquietudes de la población. La acción de articular, con las organizaciones del territorio que interpelan a la universidad, muestra que un gran número de jóvenes no llegan a la universidad, pero podrían mejorar sus condiciones de vida. Creemos que un espacio de formación y capacitación conjunta contribuirá a disminuir distancias entre saberes teóricos y prácticos. Asentando las bases para que en el intercambio de experiencias y saberes se realice de la manera más simétrica posible. Es en este sentido que consideramos que las prácticas de aprendizaje, tanto docencia, investigación y extensión, deben darse de manera contextualizada e inserta en la realidad que se atraviesa. Esto marca una diferencia, sin embargo, no es algo que caracteriza a la mayoría de las prácticas docentes, generalmente cada vez se trabaja de manera más aislada. La forma de contextualizar esas prácticas, es la integralidad donde se articulan integrando todas las funciones, docencia, investigación y extensión y en algunos casos, también la gestión institucional. Tomando como referencia las teorizaciones del profesor (Tommasino \& Rodríguez, "Tres tesis básicas sobre extensión y prácticas integrales en la Universidad de la República, bases y fundamentos", 2010) consideramos que la integralidad implica:

\section{Es en este sentido que consideramos que las prácticas de aprendizaje, tanto docencia, investigación y extensión, deben darse de manera contextualizada e inserta en la realidad que se atraviesa.}


- Integrar a la enseñanza y a la producción de conocimientos experiencias de extensión.

- Introducir a la interdisciplina en la enseñanza en sus aspectos epistemológicos (abordaje de contenidos), en la construcción de conocimiento (delimitación de objetos de estudio), y en las intervenciones que se realizan (en la resolución de problemáticas y en la conformación de equipos conformados por distintas disciplinas).

- Reconocer la voluntad transformadora de las intervenciones, en el entendido de que son los actores sociales los protagonistas directos de dichas transformaciones y no como objeto de las mismas. Este punto implica incorporar a la integralidad la participación comunitaria, el diálogo de saberes y la ética de la autonomía como eje estructurante de las intervenciones universitarias.

- Concebir de forma integral los procesos de enseñanza y aprendizaje, tanto en lo que respecta a los contenidos como a las metodologías que se utilizan. Para esto es necesario partir de una ecología de saberes, la enseñanza como proceso activo y el aprendizaje sobre la base de problemas.

- Desarrollar un enfoque territorial e intersectorial en las intervenciones y en el abordaje de problemáticas. (Tomassino, 2010: p9)

La integralidad debe ser comprendida como la articulación de actores sociales y universitarios, y no solo referir a la integración y articulación de funciones. Así concebimos a la extensión profundamente ligada al actual contexto socio- histórico, lo que la trasforma en dinámica, en diálogo con diferentes funciones universitarias. La integralidad deber ser pensada como algo que se hace y se recrea en la práctica, y no únicamente teórico conceptual.

\section{La integralidad debe ser comprendida como la articulación de actores sociales y universitarios, y no solo referir a la inte- gración y articulación de funciones.}

La extensión se aprende y se enseña en la praxis, la cual requiere ser construida junto con la sociedad en forma global, en trabajo conjunto a los movimientos y organizaciones sociales populares. Surge así un proceso dialógico y crítico que orienta el desarrollo de las otras funciones universitarias. Esta concepción implica la consolidación de las prácticas integrales y la articulación cotidiana de la investigación, la enseñanza, el aprendizaje y la extensión en la intimidad del acto educativo.

Es importante mencionar que, posicionarse desde la integralidad, implica desafíos, mezclar los tiempos, las necesidades, demandas de la universidad y de las instituciones, implica más trabajo y dedicación que prácticas aisladas; es decir que quienes optan por la integralidad 
deben tener presente que la articulación, implica más tiempo, más esfuerzo, y costos económicos. Por todo ello, se vuelve necesario contar con políticas públicas que lo respalden, o bien buscar grietas institucionales que habiliten dichas prácticas.

Sin embargo, al decir que implica más trabajo, para todas las personas involucradas, estudiantes, referentes y docente, los resultados que se obtienen son superiores a los que se puede lograr en un aula o a través de un vídeo o hasta en los mismos campos experimentales de la universidad, donde todo es adaptado para la docencia. La integralidad interpela, es la verdad, conocer estos escenarios nos enfrenta a nuevo futuro como profesionales. Cuando se avanza hacia la integralidad y se parte de la articulación, es la realidad la que marca el sentido del trabajo, y dicha realidad es indisciplinada, es imposible de enmarcarla dentro de las disciplinas, de las materias, las carreras, entonces se generan tensiones, que demandan nuevas respuestas.

\section{CONTEXTO ACTUAL DE EMERGENCIA SANITARIA: MAS IMPACTO TERRITORIAL}

La nueva dinámica social, económica y cultural impuesta por la pandemia asociada a SARS-CoV-2 ha afectado seriamente las condiciones de vida de todos los sectores de la población. Muchos de los problemas ya existentes en la sociedad, como por ejemplo la demanda alimentaria o el empleo, han crecido y afectan a más y nuevos sectores de la comunidad. Los problemas estructurales subyacentes, que van desde el acceso al agua potable hasta la alfabetización digital, condicionan las capacidades de responder apropiadamente y limitan el número de herramientas de intervención con las que se podrían mitigar los efectos de la pandemia. En este sentido, la convocatoria "Más Impacto Territorial" (+IT) pretende implementar proyectos y acciones en un contexto de fin de pandemia y post pandemia.

\section{En este sentido, la convocatoria "Más Impacto Territorial" (+IT) pretende implementar proyectos y acciones en un contexto de fin de pandemia y post pandemia.}

La propuesta se encuadra dentro de los lineamientos generales acordados en el Estatuto de la UNRC en donde se expresa que la Universidad concibe a la extensión como otra forma de construcción de conocimiento articulado al saber científico. La extensión deberá abocarse al desarrollo del conocimiento práctico, experiencial, profesional e inductivo. Este conocimiento deberá generarse de manera local, transdisciplinarmente y tener finalidades prácticas, promoviendo la 
articulación y mutuo enriquecimiento de ese tipo de conocimiento con el saber científico.

Posiblemente, la realidad del entramado social y productivo de la región en la cual se encuentra la UNRC no es demasiado diferente de aquella que refleja la estadística a nivel nacional. Sin embargo, las posibles respuestas a la creciente emergencia a nivel local, asociado con las limitaciones de los recursos disponibles, requieren de un curso de acción construido a partir de la identificación de problemas muy puntuales y capacidades instaladas en la región. Si bien existen una amplia cantidad de situaciones (problemáticas y necesidades) que deben abordarse con los recursos disponibles o mediante gestiones ante diferentes ámbitos del estado nacional, provincial y municipal, actualmente, y producto de la activa participación de la UNRC en diferentes espacios de articulación, se cuenta con un relevamiento de problemas y necesidades.

En tal sentido, y considerando que la forma más razonable de definir las problemáticas y necesidades es mediante la construcción colaborativa con (y por) actores radicados en el propio territorio donde la UNRC se despliega, particularmente se ha realizado un relevamiento conjunto en tres ámbitos con plena vigencia funcional donde la universidad tiene activa participación institucional: el Consejo Económico y Social, el Consejo Social Universitario y el Consejo Consultivo Educativo. La UNRC tiene activa participación en el Consejo Económico y Social de la ciudad de Río Cuarto, coordina el Consejo Consultivo Educativo y coordina el Consejo Social Universitario, ámbitos donde la participación de los distintos sectores de la comunidad se encuentra asegurada y validada.

\section{En tal sentido, y considerando que la forma más razonable de definir las problemáticas y necesidades es mediante la cons- trucción colaborativa con (y por) actores radicados en el propio territorio donde la UNRC se despliega, particularmente se ha realizado un relevamiento conjunto en tres ámbitos con plena vigencia funcional donde la universidad tiene activa partici- pación institucional: el Consejo Económico y Social, el Consejo Social Universitario y el Consejo Consultivo Educativo.}

En conjunto con cada consejo, se han establecido los ejes directrices generales de la presente convocatoria, los cuales deberán socializarse en los tres Consejos de vinculación institucional, como también a los Decanos de las cinco Facultades, a los consejos internos de la propia universidad (Consejo Asesor de Planeamiento y Relaciones Institucionales, Consejo Académico, Consejo de Extensión y Consejo de Ciencia y Técnica) y la Federación Universitaria de Río Cuarto a fin 
de fomentar y movilizar la plena participación de la comunidad. De esta manera se propone un proceso de generación de ideas/proyectos para el abordaje de problemas y necesidades, partiendo de una metodología de participación abierta de toda la comunidad. En consideración de los ejes directrices de problemáticas y necesidades, se iniciará un proceso que permita generar ideas/proyectos y propuestas de intervención para el abordaje de estas situaciones provenientes de la comunidad en general. En consideración a lo mencionado, el resultado del relevamiento realizado permite definir los siguientes cuatro ejes directrices para la convocatoria: educación, soberanía alimentaria, salud y economía y empleo.

El proceso de socialización y comunicación de la convocatoria es de vital importancia, toda vez que se trata de una acción que se implementa por primera vez. Se sugiere en este sentido la participación de las diferentes Facultades, claustros y estructuras de la Universidad, así como de organizaciones e instituciones del territorio. Se pretende movilizar proyectos que aborden problemáticas y necesidades de la comunidad regional, de alto impacto y capacidad de transformación, con estrategias integrales (funciones de Extensión, Investigación y Académico) y a partir de acciones intra e inter institucionales; con el fin último de dar respuestas y así fortalecer la vinculación de la UNRC con la comunidad regional.

\section{A MODO DE CIERRE}

Resulta relevante pensar lo mencionado hasta aquí desde una perspectiva temporal, a través del camino transitado, pensar los avances y logros en relación a los procesos en que se inscriben y cómo dichos procesos producen transformaciones dentro de las propias universidades, peleando por su legitimidad y ganando discusiones de legalidad y recursos.

En las prácticas integrales en donde se articulan las funciones, el rol de la extensión puede propiciar la reconfiguración, integración y renovación de todos los componentes del acto educativo. Las experiencias educativas en terreno posibilitan procesos que, a la vez, interpelan los conocimientos adquiridos en las aulas y posibilitan su mejor internalización, al vincular a los estudiantes con problemáticas sociales de su tiempo y ponerlos a trabajar junto a las comunidades que las viven, promueven procesos de transformación subjetiva y reflexión ético-crítica favorecedoras de la asunción de posicionamientos personales y colectivos críticos, responsables y solidarios.

Las prácticas integrales no pueden concretarse sin un amplio y crítico diálogo con la sociedad, sin la gestación de nuevas formas de relación que permitan que la sociedad no solo interpele a la Universidad, sino que contribuya en su reconstrucción permanente. 
Las prácticas integrales no pueden concretarse sin un amplio y crítico diálogo con la sociedad, sin la gestación de nuevas formas de relación que permitan que la sociedad no solo interpele a la Universidad, sino que contribuya en su reconstrucción permanente.

Haciendo nuestras las palabras del profesor Humberto Tomassino (Tommasino, "Las prácticas integrales en la Universidad", 2009) afirmamos en sus palabras que:

La realidad es indisciplinada. La intervención para su transformación debe ser necesariamente interdisciplinaria. Todas las disciplinas pueden y deberían estar implicadas en procesos de extensión. El diálogo interdisciplinar, originariamente académico, debe incluir, ser criticado y criticar el saber popular, conformando una red de saberes, una ecología de saberes que contribuye a la transformación participativa de la realidad.

Por último, queremos hacer hincapié en la importancia las figuras de los Consejos Sociales, como espacio centrales para potenciar el hacer, la vinculación, generar proyectos, acciones en conjunto, que interpele las condiciones establecidas, más aún en contextos totalmente extraordinario. Todo esto nos interpela y exige nuclear a docentes, no docentes, graduados y estudiantes, junto a referentes territoriales en la reflexión y acción colectiva. 


\section{BIBLIOGRAFIA}

Ministerio de Justicia y Derechos Humanos. (7 de Agosto de 1995). Ley 24.521. 18 de Noviembre de 2020, de http://servicios.infoleg.gob.ar/infoleglnternet/anexos/25000-29999/25394/texact.htm

Tommasino, H. (2009). "Las prácticas integrales en la Universidad". Santa FeArgentina: Universidad Nacional del Litoral.

Tommasino, H., \& Rodríguez, N. (2010). "Tres tesis básicas sobre extensión y prácticas integrales en la Universidad de la República, bases y fundamentos". Uruguay: Universidad de la República. Uruguay.

Universidad Nacional de Rio Cuarto. (2016). Resolución Rectoral 371/2016. 18 de Noviembre de 2020, de Universidad Barrial: https://www.unrc.edu.ar/unrc/ uniysoc/unibarrial.php

UNRC. (2009). Universidad Nacional de Rio Cuarto. 18 de Noviembre de 2020, de Resolución 322/2009: https://www.unrc.edu.ar/unrc/planeamiento/psc.php

UNRC. (2011). Universidad Nacional de Rio Cuarto. 18 de Noviembre de 2020, de Estatuto: https://www.unrc.edu.ar/unrc/tmp/docs/EstatutoUNRC.pdf

UNRC. (2014). Universidad Nacional de Rio Cuarto. de Noviembre de 2020, de https://www.unrc.edu.ar/cyt/assets/esquema_reforma_secyt.pdf

UNRC. (26 de Agosto de 2015). Universidad Nacional de Rio Cuarto. Recuperado el 18 de Noviembre de 2020, de https://www.unrc.edu.ar/unrc/n_comp.cdc?no$\mathrm{ta}=29147$

UNRC. (2020). Universidad Nacional de Rio Cuarto. Recuperado el 18 de Noviembre de 2020, de Mas Impacto Terrotorial: https://www.unrc.edu.ar/unrc/ masimpactoterritorial/

\section{Autores/as}

* Médico Veterinario | Profesor Asociado de la Universidad Nacional Río Cuarto | Especialista en Docencia Universitaria.

**Profesora de Historia

***Licenciada en Trabajo Social 